\title{
The prospects of detecting exo-planets with the Ground-based European Nulling Interferometer Experiment (GENIE)
}

\author{
R. den Hartog ${ }^{1}$, O. Absil ${ }^{2}$, P. Gondoin ${ }^{1}$, A. Stankov ${ }^{1}$, J.C. Augerau ${ }^{3}$, \\ V. Coudé du Forresto ${ }^{4}$, D. Mourard ${ }^{5}$, M. Fridlund ${ }^{1}$, P. Gitton ${ }^{6}$, \\ F. Puech ${ }^{6}$, R. Wilhelm ${ }^{6}$, M. Barrilot ${ }^{7}$, and R. Flatscher ${ }^{8}$ \\ ${ }^{1}$ Science Payloads and Advanced Concepts Office, ESA, ESTEC, Noordwijk, The Netherlands \\ email: rdhartog@rssd.esa.int \\ ${ }^{2}$ Institut d'Astrophysique et de Géophysique, Université de Liège, Belgium \\ ${ }^{3}$ Laboratoire d'Astrophysique de l'Observatoire de Grenoble, France \\ ${ }^{4}$ Université de Paris Meudon, France \\ ${ }^{5}$ Observatoire de Côte d'Azur, France \\ ${ }^{6}$ European Southern Observatory, Garching bei München, Germany \\ ${ }^{7}$ Alcatel Alenia Space, Cannes, France \\ ${ }^{8}$ EADS Astrium, Friedrichshafen, Germany
}

\begin{abstract}
The European Space Agency's Darwin and NASA's Terrestrial Planet Finder (TPF) are among the most challenging space science missions ever considered. Their principal objective is to detect Earth-like planets around nearby stars and to characterize their atmospheres. Darwin and TPF-I are currently conceived as nulling interferometers with free-flying telescopes. Within the frame of the Darwin program, the ESA and the European Southern Observatory (ESO), supported by European industries and scientific institutes, have performed two parallel Phase A studies of a ground-based nulling interferometry experiment (GENIE) at the site of ESO's Very Large Telescope Interferometer (VLTI) in Paranal, Chile. GENIE will demonstrate several key technologies required for the Darwin mission. Its science objectives include the detection and characterization of dust disks and low-mass companions around nearby stars. These studies have established detailed instrumental designs, in which GENIE will operate in the L' band around 3.8 microns as a single Bracewell nulling or constructive interferometer, using either two Auxiliary or two Unit Telescopes. The studies were supported by detailed numerical simulations which indicated the possibility of detection and low-resolution spectroscopy in nulling mode of extra-solar giant planets (EGPs) with atmospheric temperatures down to $700 \mathrm{~K}$, provided that a proper calibration of instrumental effects is applied. Detection of circumstellar exo-zodiacal (EZ) dust clouds is possible down to $0.5 \mathrm{mJy}$, with interesting prospects for the characterization of planet-forming disks.
\end{abstract}

Keywords. instrumentation: interferometers, techniques: interferometric, planetary systems, protoplanetary disks.

\section{Introduction}

GENIE, the Ground-based European Nulling Interferometry Experiment, is a collaboration between ESO and ESA, and intended for commissioning on ESOs VLTI facility in Paranal (Gondoin et al., 2005). Its objectives are to obtain experience with the design, construction and operation of a nulling interferometer, and as a preparation for the science program of ESA's DARWIN mission (Fridlund and Gondoin, 2003). In this context are foreseen the demonstration of deep nulling with a stellar light suppression more than 

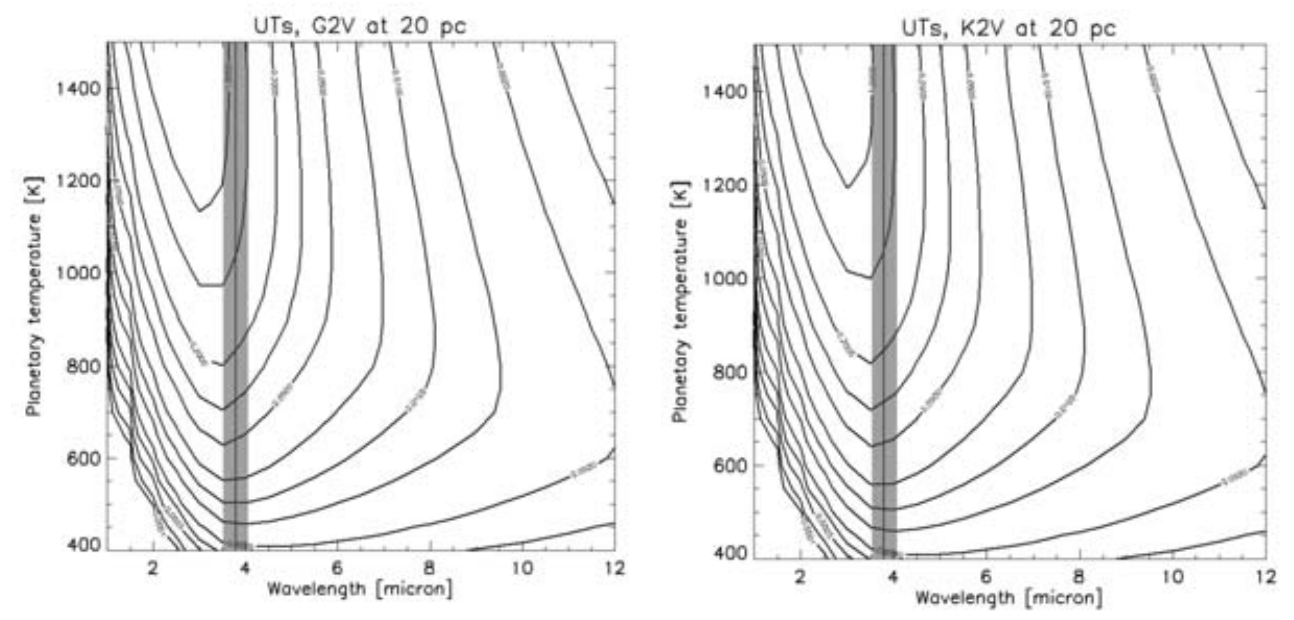

Figure 1. Wavelength trade-off on the basis of (maximum) S/N ratio (obtainable in one second) for the detection of EGPs, for two stellar types. The plots show iso-SNR contours. The SNR peaks in the upper left corner of the diagrams. In particular for lower planetary temperatures, the SNR peaks near the L' band (indicated by the grey band) and then decreases towards lower wavelengths as the star/planet contrast deteriorates, and towards higher wavelengths as the shot noise from the IR background increases.

a factor 1000, and the screening of targets of the Darwin catalogue (Eiroa et al., 2003, Kaltenegger et al., 2005) for the presence of zodiacal emission, and, when possible, the presence of EGPs. EZ clouds with 20 times the density of the solar cloud may seriously hamper the detection of Earth-like planets, and the presence of giant planets in orbits inside the inner habitable zone (HZ) radius may either indicate the absence of Earth-like planets in the $\mathrm{HZ}$, or hamper their detection if present. Paranal provides an excellent vantage point with access to $\approx 90 \%$ of the targets in the Darwin catalogue. An instrument that is capable of detecting faint dust disks or giant planets within a fraction of an arcsec from a star which is 3 to 4 orders of magnitude brighter, is obviously useful to other lines of research as well (Gondoin et al., 2005); GENIE is therefore foreseen to become a general user instrument at Paranal, with a constructive interferometry mode in addition to its nulling capabilities.

During 2004, GENIE was the subject of a parallel Phase A study by two industrial consortia, one led by EADS Astrium Space (Flatscher et al., 2004) and the other by Alcatel Alenia Space (Barillot et al., 2004). Both consortia were asked to select two interferometric concepts, based on VLTI infrastructure and instrumentation specifications, to provide detailed optical and mechanical designs for these concepts, and to assess their performance. Although the instrumental designs forwarded by the two consortia differed in many details, a remarkable agreement was found in their conclusions. The only two feasible concepts were found to be a single Bracewell nulling interferometer, and a splitpupil double Bracewell (i.e. a Keck nuller, see Mennesson et al., 2005, or Serabyn et al., 2004). Both concepts will work with any two UT's or two AT's. The L'-band was selected as the optimal waveband for the detection of both EZ dust and giant exo-planets. An illustration of the wavelength trade-off in terms of signal-to-noise ratios (SNR) for EGP detection is shown in Fig. 1. The performance predictions were also remarkably similar, despite differences in the set-up of the control loops.

During the studies it also became clear that GENIE is depending on a strict realization of the VLTI specifications of its noise environment. A driver for the specifications of the 
Table 1. Simulated GENIE nulling performance over 100 sec. observations, for a range of atmospheric conditions.

\begin{tabular}{ccc}
\hline & worst case & best case \\
\hline Piston & $17 \mathrm{~nm} @ 20 \mathrm{kHz}$ & $6.2 \mathrm{~nm} @ 13 \mathrm{kHz}$ \\
Dispersion & $17.5 \mathrm{~nm} @ 200 \mathrm{~Hz}$ & $4.5 \mathrm{~nm} @ 300 \mathrm{~Hz}$ \\
Intensity & $4 \% @ 1 \mathrm{kHz}$ & $4 \% @ 1 \mathrm{kHz}$ \\
\hline Mean total nulling & $9.7 \times 10^{-4}$ & $6.2 \times 10^{-4}$ \\
Mean instrumental nulling & $5.0 \times 10^{-4}$ & $1.5 \times 10^{-4}$ \\
RMS instrumental nulling & $5.8 \times 10^{-4}$ & $1.6 \times 10^{-4}$ \\
\hline Zodi level for SNR=5 (raw) & 1800 & 530 \\
Zodi level for SNR=5 (calibrated) & 56 & 34 \\
\hline
\end{tabular}

Genie control loop for the stabilization of the optical path difference (OPD) between the two beams turned out to be a $750 \mathrm{~Hz}$ resonance of the bimorph mirror used in the VLTI adaptive optics system MACAO. Also, the level of differential polarization between two arms, which is currently not known in sufficient detail, is subject to rather stringent requirements. The two consortia have specified detailed tests to resolve this point, which were endorsed by the $123^{\text {rd }}$ meeting of ESA's Astronomical Working Group (AWG), pointing out that the success of the Darwin mission depends critically on a tight control of all polarization effects.

\section{Simulated detections}

The current analysis of the GENIE science performance is based on computations with the GENIE science simulation software package "GENIEsim" (Absil et al., 2003 and 2005), which has been updated with the results from the two parallel Phase A studies, and the latest information supplied by ESO about VLTI performance.

\subsection{Geniesim}

GENIEsim models the full history of the science photons. The generation in the target system by star or planet is modelled with black bodies, and by the EZ dust cloud through a deprojection of the COBE/DIRBE Interplanetary Cloud Model (Kelsall et al., 1998) with the zodipic package by Kuchner, 2001. The description of the turbulent aspects of the atmosphere are based on the standard Kolmogorov model combined with the Taylor hypothesis and include atmospheric OPD, (water-vapour) dispersion, Strehl, tip/tilt, scintillation and IR background fluctuations. The modelling of the VLTI environment is based on ESO specifications of the MACAO adaptive optics, the PRIMA fringe sensing units, the delay line and, optionally, the IRIS tip/tilt sensing and correction, and includes control loop models as well as additional noise from bimorph-induced OPD and tunnelinduced tip/tilt, plus an IR background contribution from $\approx 20$ mirror surfaces. The GENIE instrument model includes the basic functions of wavefront filtering with a singlemode fiber, nulling with a modified Mach-Zender beam-combiner and detection with a state-of-the-art IR focal-plane array, plus the corrections by closed control-loop systems for OPD, dispersion, intensity mismatch and, optionally, tip/tilt.

\subsection{Control loop performance}

The main output of GENIEsim is a time sequence of detector readings, which can be converted into nulling ratios or SNR's by keeping a detailed administration of the noise 
Table 2. Properties of seven short period EGP systems accessible from Paranal.

\begin{tabular}{|c|c|c|c|c|c|c|c|c|c|c|c|c|c|c|}
\hline \multirow{3}{*}{ target $\quad s$} & \multicolumn{2}{|l|}{ tar } & \multicolumn{4}{|c|}{ planet } & \multicolumn{8}{|c|}{ signal to noise ratio } \\
\hline & sp. type & dist. & $\mathrm{M} \sin i$ & per. & $a$ & temp. & $B_{\text {proj }}$ & in $4 \mathrm{~h}$ & & in $\mathrm{spe}$ & ectra & al che & annel & \\
\hline & & {$[\mathrm{pc}]$} & {$\left[M_{\mathrm{jup}}\right]$} & [day] & {$[\mathrm{AU}]$} & & & & 4 & 8 & 16 & 32 & 64 & 128 \\
\hline HD 73256 & G8 V & 36.5 & 1.87 & 2.55 & 0.037 & 1200 & 129.5 & 6.4 & 5.0 & 4.1 & 3.1 & 2.2 & 1.4 & 0.7 \\
\hline HD 83443 & $\mathrm{~K} 0 \mathrm{~V}$ & 43.5 & 0.41 & 2.99 & 0.035 & 1117 & 128.6 & 4.4 & 2.5 & 1.8 & 1.3 & 0.9 & 0.5 & 0.3 \\
\hline HD 179949 & $\mathrm{~F} 8 \mathrm{~V}$ & 27.0 & 0.98 & 3.09 & 0.045 & 1383 & 102.4 & 4.4 & 4.4 & 4.3 & 4.3 & 4.1 & 3.7 & 2.6 \\
\hline Tau Boo & F7 V & 15.0 & 4.13 & 3.31 & 0.047 & 1388 & 60.9 & 3.4 & 3.4 & 3.4 & 3.4 & 3.4 & 3.4 & 3.2 \\
\hline HD 75289 & G0 V & 28.9 & 0.42 & 3.51 & 0.047 & 1265 & 126.7 & 4.5 & 4.5 & 4.4 & 4.3 & 4.1 & 3.5 & 2.4 \\
\hline HD 162020 & $\mathrm{~K} 2 \mathrm{~V}$ & 31.3 & 13.8 & 8.42 & 0.072 & 784 & 129.0 & 3.9 & 3.1 & 2.5 & 1.9 & 1.4 & 0.8 & 0.4 \\
\hline HD 130322 & K0 V & 30. & 1.08 & 10.7 & 0.088 & 724 & 123.6 & 3.8 & 2.7 & 2.0 & 1.5 & 1.0 & 0.6 & 0.3 \\
\hline
\end{tabular}

sources. Table 1 shows the expected nulling performance of GENIE during 100 sec. observations, taking into account all turbulence- and VLTI-induced disturbances. The performances of the control loops are summarized together with their repetition frequencies under both pessimistic and optimistic atmospheric conditions (see Absil et al., 2005 for further details). The mean total nulling ratio includes contributions from both the geometric leakage (due to the fact that the stellar disk is resolved by the nulling interferometer) and instrumental leakage (due to disturbance-driven null floor and intensity matching excursions), and is computed as the ratio between the total input and output stellar fluxes (from both telescopes). The next row gives the standard deviation of the instrumental nulling ratio for this $100 \mathrm{sec}$ observation block, a number governing the (im-)possibility of the direct detection of EZ clouds and planets. The last two rows give the density of EZ dust, measured in solar units or zodis, that can be detected to an SNR of 5 around a Sun-like at 20 pc in 1 hour in the full L' band with two UTs. The simulation indicates that with the expected null stability it will be possible to detect EZ light to a useful level only after calibration.

\subsection{Calibration procedure}

The calibration scheme consists of estimation and subtraction of the three main noise sources (IR background, geometrical and instrumental stellar leakage) are estimated and subtracted from the signal, leaving only the planet signal. The IR background can be estimated either with two off-axis fibers that just fit in the field of view adjacent to the on-axis science fiber, or by applying a split-pupil design.

The geometrical leakage is given by $G=(\pi / 2)^{2}\left(\theta_{\star} B / \lambda\right)^{2}$, and the accuracy of its estimate is limited mainly by the accuracy with which the stellar angular radius $\theta_{\star}$ can be derived. For the closest stars direct interferometric measurements will provide accuracies $\Delta \theta_{\star} / \theta_{\star}<0.5 \%$. For more distant stars such accuracies are possible using empirical relations between (limb-darkening corrected) angular diameters and e.g. effective temperatures (Kervalla et al., 2004). Such methods do require apparent magnitudes to be known better than $0.02 \mathrm{mag}$, thus calling for an additional photometry programme. In Table 1 a diameter precision of $0.5 \%$ is considered best case, and $1 \%$ worst case.

The instrumental leakage will be derived from observations of calibrator stars. Calibrator stars should exhibit $a$ ) negligible emission from dust or planets, $b$ ) be at least as bright as the target in order to feed the control loops with the same photon flux, $c$ ) be located in the same part of the sky to ensure similar atmospheric conditions, and $d$ ) have radii that are either small enough to ignore geometrical leakage, or large enough to allow accurately estimates. Distant late-type giants (typically K III) appear the best 


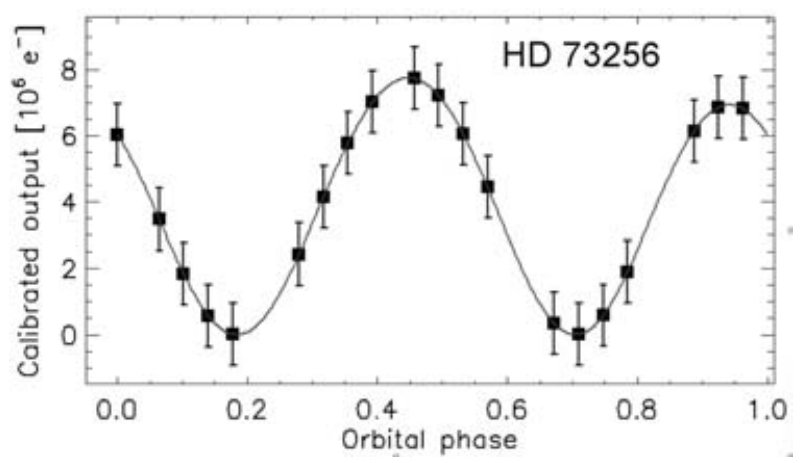

Figure 2. Illustration of the expected results after 20 consecutive nights of observation on HD 73256 , assuming a sub-optimal estimate of the stellar diameter $\left(\Delta \theta_{\star} / \theta_{\star}=1 \%\right)$.

candidates. Several catalogues have already been compiled for these type of photometric calibrators (Mérand et al., 2005; Cohen et al., 1999).

\section{Detection and spectroscopy of EGP}

The detections of seven short-period EGP were simulated and the results are summarized in Table 2. For each target this Table lists the relevant properties of star and planet. The planetary temperatures were derived assuming simple radiation equilibrium, with a planetary Bond albedo of 0.34 . All planetary radii were taken to be 1.2 Rjup, the median of 4 transition measurements (cf. Burrows et al., 2004). The simulations assume observations with 2 UT's under standard Paranal atmospheric conditions, a planet crossing a maximum of the transmission map, and a stellar diameter accuracy of $1 \%$. All listed EGP can be detected with a SNR better than 3 in 4 hours of integration. If a series of observations during consecutive nights are combined, as illustrated in Fig. 2, the SNR per observation may be lower and still allow sufficient confidence in the planetary detection. Since the SNR is dominated by the calibration accuracy of the geometrical and instrumental leakage, the L'-band can in most cases be subdivided in several spectral bands without suffering a corresponding decrease in SNR (until the read-out noise or other shot noise terms start to dominate again). Detection and low-resolution spectroscopy $(R \approx$ 100) of EGP's with temperatures as low as $700 \mathrm{~K}$ ('warm Jupiters') appear feasible.

\section{Detection of dust disks and planetary formation}

Based on GENIEsim simulations, Fig. 3 shows the expected distribution of levels of EZ emission around Darwin target stars, which are detectable at a SNR of 5 after 1 hour of integration with two UT's, and after application of a calibration scheme as outlined above. The EZ emission is measured along the ordinate in units of Local Zodiacal emission (zodi), and along the abscissa in mJy. The absolute detection limit appears to be roughly $0.5 \mathrm{mJy}$, and not strongly dependent on stellar type. For cooler stars (which have cooler dust disks, the dust needs to be denser for a detection above the SNR limit. The limits to which EZ dust clouds can be detected in solar units are 30 zodi for F stars, 40 zodi for $\mathrm{G}$ stars and 60 zodi for $\mathrm{K}$ stars.

Absil et al. (2005) have shown that innovative measurements on debris disks are possible using GENIE with the large range of baselines possible with the AT's. Figure 4 shows the nulling ratio (measured as the ratio between nulled and constructive output of the beam combiner) as a function of baseline for various models of the debris disk 


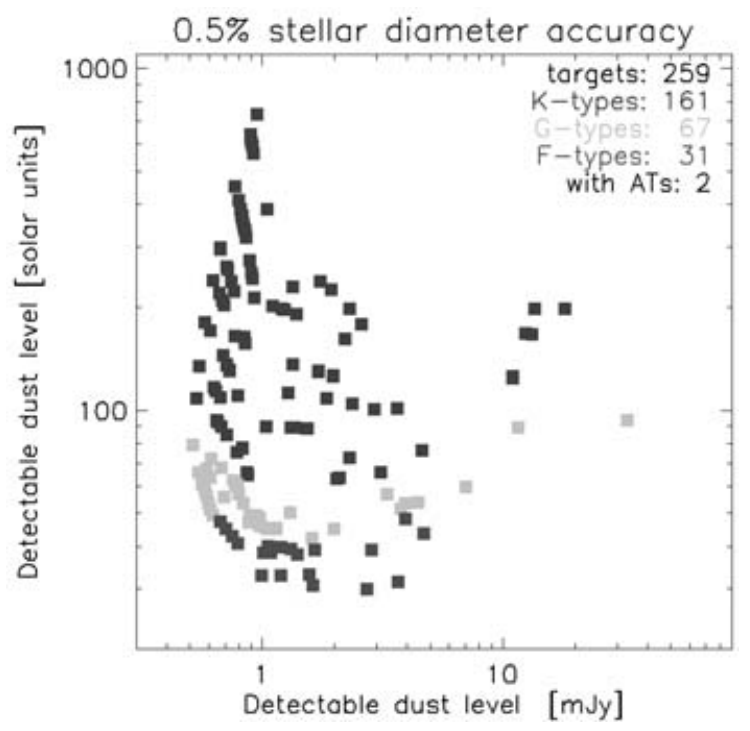

Figure 3. Dust detection limits for Darwin catalogue stars.

around $\zeta$ Lep (cf. Augerau et al., 1999). The observations were calibrated using $\eta$ Lep, a nearby $\mathrm{F} 1 \mathrm{~V}$. The nulling ratio is a function of the sublimation radius, the radius inside which dust grains are destroyed. The sublimation temperature of the grains depends on their size and composition. Cold coagulation grains are more porous than silicates, and therefore less efficient in re-emitting thermal radiation. Hence a larger density of porous grains is needed to produce the same mid-IR excess, which results in an enhanced L'-band flux, as is seen in Fig. 4a. Smaller grains are more easily destroyed than larger grains, hence for smaller grains the sublimation radius lies further out. The first maximum of the transmission map will coincide with the sublimation radius for smaller baselines, as can be seen in Fig. 4b. These figures illustrate that important clues about the structure and composition of planet-forming disks can be obtained with a fairly straightforward set of nulling experiments.
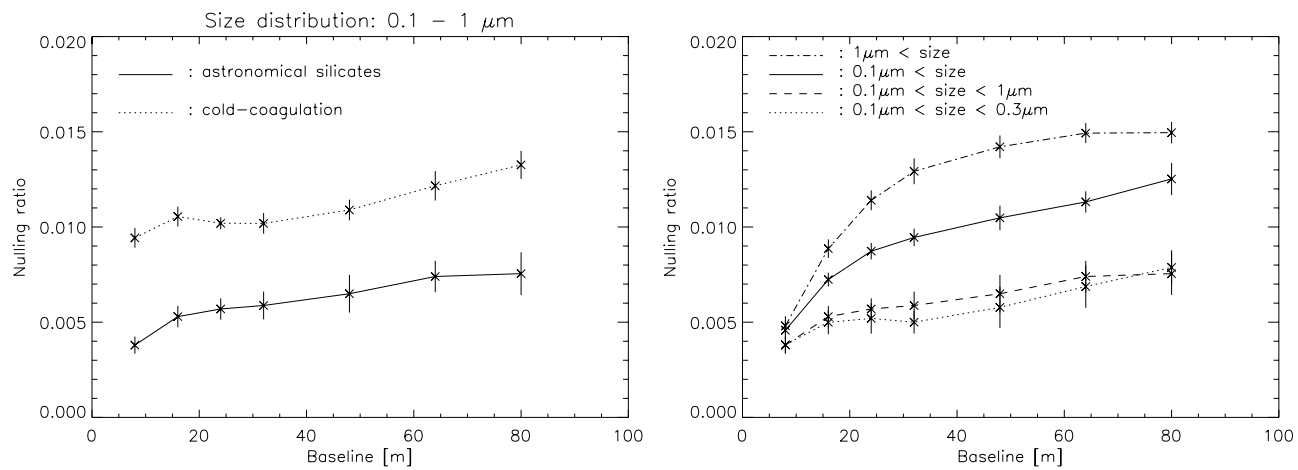

Figure 4. Nulling ratio as a function of baseline for dust disks with, respectively, different grain composition, and different ranges of silicate grain sizes. 


\section{References}

Absil, O., den Hartog, R., and Gondoin, P., 2003, in: M. Fridlund and T. Henning (eds.) Proc. of Conf. on "Towards Other Earths: Darwin / TPF and the Search for Extrasolar Terrestrial Planets (Heidelberg), ESA SP-539, 317

Absil, A., den Hartog, R., et al., 2005, A\&A in press

Augereau, J.-C., Lagrange, A.-M., et al., 1999, A $\mathscr{E} A$ 348, 557

Barillot, M., Ruilier, C., et al., 2004, Alcatel final presentation of GENIE Phase A study to ESA

Burrows, A., Hubeny, I., Hubbard W. et al., 2004, ApJ 610, L53-56

Cohen, M., Walker, R. G., Carter, B., Hammersley, P. L., Kidger, M. R., and Noguchi, K., 1999, AJ 117,1864

Coudé du Foresto, V., Absil, A., Barillot, M., Swain, M., 2005 These Proceedings

Eiroa, C., Fridlund, M. and Kaltenegger, L., 2003, in: M. Fridlund and T. Henning (eds.) Proc. of Conf. on "Towards Other Earths: Darwin / TPF and the Search for Extrasolar Terrestrial Planets (Heidelberg), ESA SP-539, 403

Flatscher, R., Wallner, O., Ergenzinger, K., et al., 2004, Astrium final presentation of GENIE Phase A study to ESA

Fridlund, M., and Gondoin, P., 2003, Proc. SPIE 4852, 394

Gondoin, P., den Hartog, R., et al. 2005, in: A. Chelli and F. Delplancke (eds.), Proc. of ESO Workshop on The Power of Optical/IR Interferometry: 2nd Generation VLTI Instrumentation, (Heidelberg: Springer), in press

Kaltenegger, L., Eiroa, C., Stankov, A. and Fridlund, M., 2005, These Proceedings

Kelsall, T., Weiland, J.L., Franz, B.A., et al., 1998, ApJ 508, 44

Kervella, P., Thevenin, F., Segransan, D., et al., 2003, A\&BA 404, 1087

Kuchner, M., 2001, IDL zodipic package, see http://www.astro.princeton.edu/ mkuchner

Mennesson, B., et al., 2005 These Proceedings

Mérand, A., Bordé, P., and Coudé du Foresto, V., 2005, A\&SA 433, 1155

Serabyn, E. et al., 2004, Proc. SPIE 5491, 806 

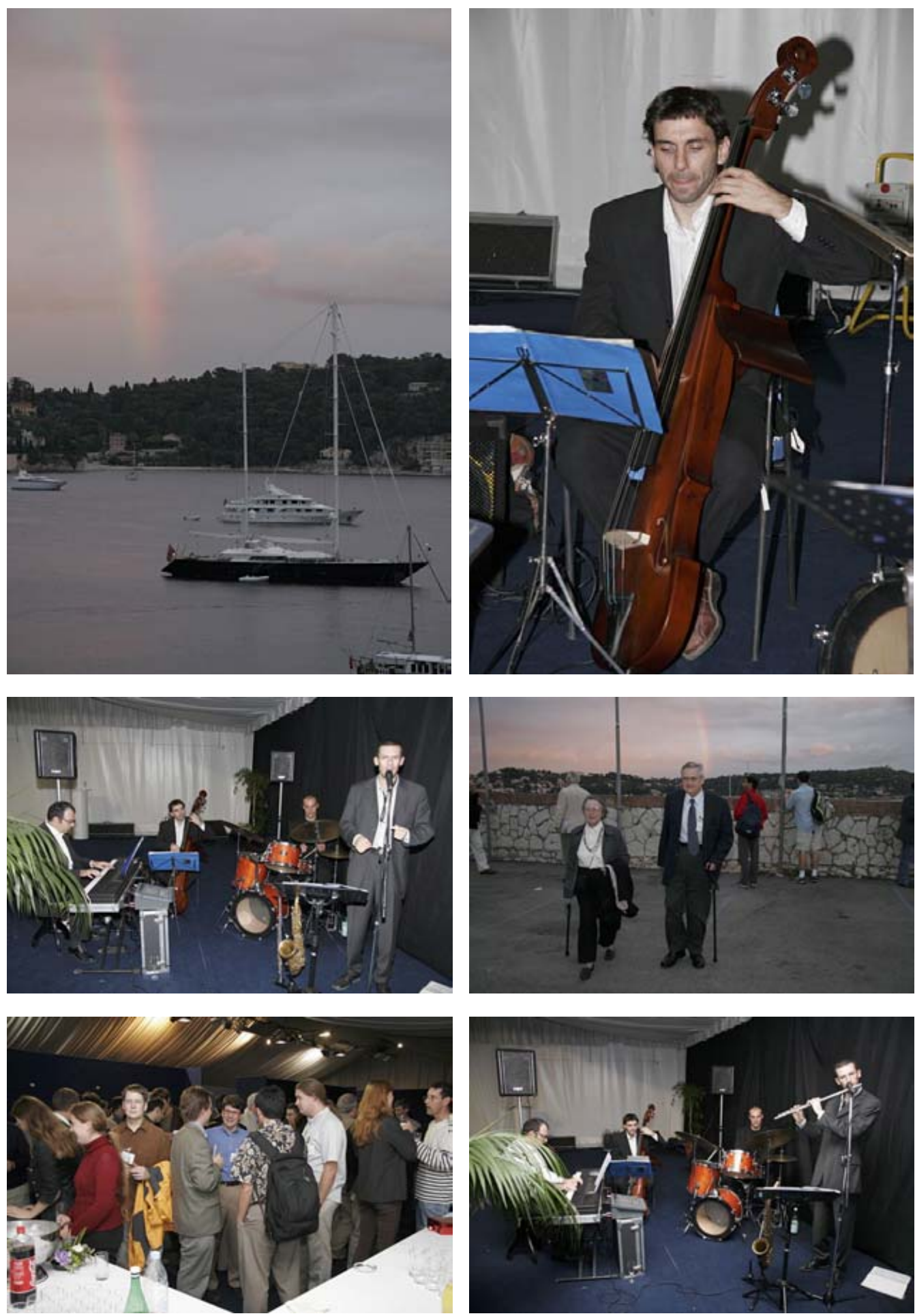

All photographs: Laurent Thareau [1.thareau@free.fr]. 